OPEN ACCESS

\section{Possible experience: From Boole to Bell}

To cite this article: K. Hess et al 2009 EPL 8760007

View the article online for updates and enhancements.
Related content

- $\frac{\text { Hidden assumptions in the derivation of }}{\text { the theorem of Bell }}$
Karl Hess, Hans De Raedt and Kristel
Michielsen

Michielsen

Modeling experiments using quantum and Kolmogorov probability

Karl Hess

Mathematical and physical meaning of the Bell inequalities

\section{Recent citations}

- Simultaneous measurement of non-
$\frac{\text { commuting observables in entangled }}{\text { systems }}$
Erik F. G. van Heusden and Theodorus M.
Nieuwenhuizen
- Closing the Door on Quantum Nonlocality
Marian Kupczynski
- Quantum mechanics and modeling of
physical reality
Marian Kupczynski




\title{
Possible experience: From Boole to Bell
}

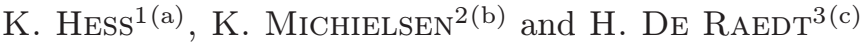 \\ ${ }^{1}$ Beckman Institute, Department of Electrical and Computer Engineering and Department of Physics, \\ University of Illinois - Urbana, IL 61801, USA \\ ${ }^{2}$ Institute for Advanced Simulation, Jülich Supercomputing Centre, Research Centre Jülich \\ D-52425 Jülich, Germany, EU \\ ${ }^{3}$ Department of Applied Physics, Zernike Institute for Advanced Materials, University of Groningen \\ Nijenborgh 4, NL-9747 AG Groningen, The Netherlands, EU
}

received 1 July 2009; accepted in final form 11 September 2009 published online 9 October 2009

PACS 03.65.Ud - Entanglement and quantum nonlocality (e.g. EPR paradox, Bell's inequalities, GHZ states, etc.)

PACS 03.65.Ta - Foundations of quantum mechanics; measurement theory

PACS 03.65.-w-Quantum mechanics

\begin{abstract}
Mainstream interpretations of quantum theory maintain that violations of the Bell inequalities deny at least either realism or Einstein locality. Here we investigate the premises of the Bell-type inequalities by returning to earlier inequalities presented by Boole and the findings of Vorob'ev as related to these inequalities. These findings together with a space-time generalization of Boole's elements of logic lead us to a completely transparent Einstein local counterexample from everyday life that violates certain variations of the Bell inequalities. We show that the counterexample suggests an interpretation of the Born rule as a pre-measure of probability that can be transformed into a Kolmogorov probability measure by certain Einstein local space-time characterizations of the involved random variables.
\end{abstract}

Copyright (c) EPLA, 2009

Introduction. - We discuss models of EinsteinPodolsky-Rosen-Bohm type $[1,2]$ of experiments as used by John Bell [3] when presenting his celebrated inequalities. These experiments result in outcomes of two spin-values \pm 1 (in units of $\hbar / 2$ ) that in turn depend on certain magnet settings $\mathbf{a}, \mathbf{b}, \mathbf{c} \ldots$ and have been linked to two-valued functions $A_{\mathbf{a}}(\cdot), A_{\mathbf{b}}(\cdot), A_{\mathbf{c}}(\cdot)= \pm 1$ by Bell and followers. Here $(\cdot)$ stands for the dependency on some element of a set of mathematical representations of elements of reality that do not depend on the magnet settings $\mathbf{a}, \mathbf{b}, \mathbf{c} \ldots$. This latter fact of independence from magnet settings was deduced by Bell from considerations of Einstein locality and the (physically unjustified) assumption that the elements of reality emanate exclusively from a distant source and not from the measurement equipment (including the magnets). There are numerous inequalities, delineated in the physics literature that are related to Bell's functions $A_{\mathbf{a}}(\cdot), \ldots$. These inequalities were first derived by Boole [4] in a much more general context. Here we discuss

\footnotetext{
(a) E-mail: k-hess@illinois .edu

(b) E-mail: k.michielsen@fz-juelich.de

(c) E-mail: h.a.de.raedt@rug.nl
}

mainly a variation of the inequalities as published by Leggett and Garg [5], for which we also have developed a transparent counterexample. More complex counterexamples have been developed in the past for the more elaborate inequalities [6] but have remained largely unappreciated because of their lack of transparency. Nevertheless, strong movements critical of Bell's approach continue to grow as demonstrated by contributions of Accardi, Fine, Rastal, Khrennikov, Nieuwenhuizen and many others [7-11]. Here, however, we do not refer to non-Kolmogorovian approaches [11] and we like to stress that we also do not invoke detector inefficiencies or anything related to fair sampling [12]. Instead, our counterexample is based on a more complete characterization of Boole's logical elements.

The Leggett-Garg inequality reads

$$
A_{\mathbf{a}}(\cdot) A_{\mathbf{b}}(\cdot)+A_{\mathbf{a}}(\cdot) A_{\mathbf{c}}(\cdot)+A_{\mathbf{b}}(\cdot) A_{\mathbf{c}}(\cdot) \geqslant-1 .
$$

Inserting all possible values of \pm 1 for the functions $A(\cdot)$ shows the correctness of this inequality. Because measurement outcomes of Einstein-Podolsky-Rosen (EPR) experiments [13] (that are closely related to such two-valued functions $A(\cdot))$ do violate this inequality, it is commonly 
concluded that either $(\cdot)$ cannot stand for any element of reality and one must therefore abandon realism or if it stands for an element of reality it must depend on the magnet settings and thus violate Einstein locality. There are, however, two important questions that have never been answered satisfactorily. If $(\cdot)$ stands for an element of reality, why does it have to appear identically for the three magnet setting pairs? If, on the other hand, $(\cdot)$ is just seen as a random variable, why do the functions $A$ not also depend on a measurement time label, as they are introduced in the theory of stochastic processes [14]? We give below a clear answer to these questions by means of our counterexample but discuss first the different views taken in the well-established probability theories of Boole and Kolmogorov as well as quantum mechanical "probability" as introduced by the Born rule.

The probability theory of Boole and its generalization and perfection by Kolmogorov reduce the actual experiments to logical abstractions and establish a one to one correspondence between the experiments and these abstractions. For the case that interests us we have only two possible experimental outcomes denoted by \pm 1 (or equivalently 0,1 or true and false). "Probability" is defined by Boole and Kolmogorov by imposing a measure (a real number of the interval $[0,1]$ ) onto these elements that is consistent with the experimental factors related to both the single logical abstractions as well as the whole set of these abstractions. This is the hallmark of modern probability theory and emphasizes the relation to set theory.

The one to one correspondence of mathematical abstractions to actual experiments and a measure on the set of these abstractions are both necessary to give meaning to the word probability in a set-theoretic sense. The less familiar reader is encouraged to look at these definitions in the original work of Boole [4] or, for the Kolmogorov framework, in textbooks such as [15]. For such a model to make general sense in all experimental situations, we must assume that (1) a given and well-defined logical element representing an experimental outcome or, in the language of Kolmogorov, an elementary event will occur with the same probability measure throughout all experiments and that (2) the physical characterization of the logical elements of Boole (elementary events of Kolmogorov) is consistent and complete throughout the experimental sequence.

This requirement for the description of experiments by mathematical and logical abstractions that represent a "truth" throughout an experimental sequence, brings us back to the fundamental statement of Plato's logic: " $P$ aut non $P$ tertium non datur" and goes to the heart of discussions related to questions such as "does the moon shine when I am not looking?". The sentence "The moon shines" is, in general too ill defined to be identified with a logical variable, say $B$ that assumes a value +1 if the moon shines and -1 if it does not. Throughout any reasonably general experimental sequence that lasts for a certain duration, the moon may or may not shine at certain different places and $B$ will therefore assume a variety of values at these different places. Correspondingly a certain outcome of $B$ cannot stand for the same mathematical abstraction that describes facts at different locations. If we wish to associate with $B$ a certain truth or logical expression that is valid everywhere and throughout the experimental sequence we need to introduce some generalized coordinates and formulate a more precise statement such as "the moon was shining in Monte Carlo at a certain date and time". In connection with general science experiments we need to note that a statement about experimental outcomes often may make no sense whatsoever without the introduction of a coordinate system.

Therefore, we propose the use of the space-time of special relativity to complete the characterization of Boole's logical elements and Kolmogorov's elementary events. We assume that only this completion can lead to true-false or other binary statements that are always and everywhere valid even in very complex one to one correspondences of mathematical abstractions with actual experiments.

We can, as a simple example, have a number of coins and measure the outcome of coin-tosses at certain given space-time coordinates. The coins may contain some magnetic material and there may be hidden magnets with settings $\mathbf{a}, \mathbf{b}, \mathbf{c}$ that co-determine a probability to measure head or tail for the given coins at the given space-time coordinates. For given magnet settings and space-time coordinates of the coins we have then certain outcomes that form a sample space and certain probabilities for the outcomes that together with the sample space form a probability space [15]. If we do not label the coins by their correct space-time coordinates then we may have, for example, different magnet settings applying to the same coin and therefore may have different probabilities for the outcomes of the coin-toss which may lead to confusion and contradictions.

Quantum theory uses a variation of probability theory by invoking a wave function $\psi$ that does not have a direct physical interpretation but does correspond to a certain experimental procedure of preparation. The settings of the macroscopic measurement equipment can be chosen at will and the measurements may be performed involving detection of particles that involve a space-time description through the many-body Hamiltonian and wave function $\psi$. The "probability" to measure a particle by the given equipment with given setting is then related by Born's interpretation to the absolute square (a positive number) of the wave function that thus assigns a positive number to an event once the actual type of measurement is chosen. This assignment, however, cannot yet be regarded as a probability measure in the spirit of Boole or in terms of Kolmogorov's definitions because there is no assignment made at this point for a sample space, i.e. a space of all possible outcomes and corresponding elementary events or logical elements. The Born rule appears thus as a pre-measure that may be expanded to a full Kolmogorov 
probability measure only after all experiments of a sequence are chosen i.e. once all macroscopic equipment configurations of measurements and all possible outcomes (data) are fully determined. If we desire to create a Kolmogorov frame model based on Born's rule, then the actual choice of random variables may also necessitate the introduction of one or more stochastic processes in order to include time coordinates that are otherwise not included in the Kolmogorov framework. Even this advanced procedure as described, e.g., in [14] leaves us with the vexing problem of determination which mathematical abstractions (elementary events of Kolmogorov or logical elements of Boole) correspond to the different actual experiments.

For example, assume that one measures correlated pairs of spin- $1 / 2$ particles with magnet settings $\mathbf{a}, \mathbf{b}$ and characterizes the dichotomic outcomes for the $\mathbf{a}, \mathbf{b}$ settings by the variables $A_{\mathbf{a}}, A_{\mathbf{b}}$. Further assume that in another set of measurements we measure with magnet settings a, $\mathbf{c}$. Can we then denote the corresponding variables for the outcomes by $A_{\mathbf{a}}, A_{\mathbf{c}}$ ? Recall that, in this second case, we measure the " $A_{\mathbf{a}}$ " outcomes corresponding to the $\mathbf{c}$ setting (in the other wing of the experiment) at different spacetime coordinates and with different correlated pairs as compared to the first case " $A_{\mathbf{a}}$ " outcomes that correspond to the original $\mathbf{b}$ setting. Is it then permitted to use the same dichotomic variable or logical element as used for the b setting?

Because a sample space and single outcomes are not included into considerations of quantum theory, this theory does not answer the above question. The Born rule per se does therefore not provide probabilities in the sense of Boole or Kolmogorov but can only lead to a probability once a one to one assignment of mathematical elements and experimental outcomes is made and a measure for the whole space of possible outcomes, the whole sample space, is introduced. This cannot be accomplished by normalizing a given wave function because that normalization refers only to a single preparation and measurement of a much more elaborate sequence of experiments. However, it is clear that for measurements with a given macroscopic setting and a fixed method of preparation, sample spaces can always be created and that such a sample space of measurement outcomes together with the probabilities from Born's rule forms then also a probability space à la Kolmogorov for a given setting as outlined in texts such as [14]. Nevertheless, for different and particularly for incompatible experiments and for a given characterization of functions or random variables e.g. by magnet settings only, such a probability space may not exist.

As we will see in our counterexample this non-existence depends crucially on the one-to-one correspondence of the experimental outcomes to their mathematical idealizations be they elements of Boolean logic or elementary events in the framework of Kolmogorov.

Many mathematical papers on probability theory simply start with the phrase "given a Kolmogorov probability space ...". It is, however, well known and has been particularly well pointed out by Vorob'ev [16] that there are cases in which a Kolmogorov probability space does not exist. In particular, there exist numerous classical experiments that subject to certain characterizations by simple settings, cannot be described on one probability space in a logically consistent way. Take, for example, certain physical experiments that can be described by Stochastic Processes. Examples are Brownian motion or stock market and exchange rate fluctuations. It is plausible that such different processes may not be describable by a single stochastic process but are described rather by different ones. It is less known but has been shown in great detail that even very slight changes in experiments may require the use of different stochastic processes for their description and that this is true also for EPR-type experiments.

It is the purpose of this paper to show that Born's rule defines a pre-probability measure that only then can be turned into a Kolmogorov (or Boole) probability if a logically consistent one to one correspondence between experimental outcomes and mathematical abstractions is or can be made. We also show that such one to one correspondence can always be made for the known EPR experiments by completing the characterization of the mathematical symbols describing the functions $A$ of Bell by use of space-time indices that relativity theory provides us with. Indices related to influences at a distance would also accomplish the same goal of obtaining a consistent probability measure à la Kolmogorov from Born's rule but do not appear to be necessary.

Games with symptoms and patients: from Boole to Bell. - As mentioned, the early definitions of probability by Boole were related to a one to one correspondence that Boole established between actual experiments and idealizations of them through elements of logic with two possible outcomes. His view gave the concept of probability precision in its relation to sets of experiments and this precision is expressed by Boole's discussion of probabilities as related to possible experience. These discussions can be best explained by an example that also shows the role of space-time coordinates in the characterization of variables related to probability theory. We discuss first this example that has its origins in the works of Boole and also Vorob'ev and relates to the work of Bell inasmuch as it can be used as a counterexample to Bell's conclusions related to nonlocality. Then we return to the more general discussions of probability in quantum theory.

Consider a certain disease that strikes persons in different ways depending on circumstances such as place of birth and place of residence etc. Assume that we deal with one set of patients that are born in Africa (subscript a), in Asia (subscript b) and in Europe (subscript c). Assume further that doctors are assembling information about the disease altogether in the three cities Lille, Lyon and Paris, all in France. The doctors are careful and perform the 
investigations on randomly chosen but identical dates. The patients are denoted by the symbol $A_{\mathbf{o}}^{l}(n)$ where $\mathbf{o}=\mathbf{a}, \mathbf{b}, \mathbf{c}$ depending on the birthplace of the patient, $l=1,2,3$ depending on where the doctor gathered information 1 designating Lille, 2 Lyon and 3 Paris respectively, and $n=1,2,3, \ldots, N$ denotes just a given random day of the examination. The doctors assign a value $A= \pm 1$ to each patient; $A=+1$ if the patients show a certain symptom and $A=-1$ if they do not.

The first variation of this investigation of the disease is performed as follows. The doctor in Lille examines all patients of type a, the doctor in Lyon all patients of type b and the doctor in Paris all patients of type c. On any given day of examination (of precisely one patient for each doctor and day) they write down their diagnosis and then, after many exams, concatenate the results and form the following sum of pair-products of exam outcomes at a given date described by $n$ :

$$
\Gamma(n)=A_{\mathbf{a}}^{1}(n) A_{\mathbf{b}}^{2}(n)+A_{\mathbf{a}}^{1}(n) A_{\mathbf{c}}^{3}(n)+A_{\mathbf{b}}^{2}(n) A_{\mathbf{c}}^{3}(n) .
$$

Boole noted now that

$$
\Gamma(n) \geqslant-1
$$

which can be found by inserting all possible values for the patient outcomes summed in eq. (2). For the average (denoted by $\langle$.$\rangle ) over all examinations we have then also:$

$$
\Gamma=\langle\Gamma(n)\rangle=\frac{1}{N} \sum_{n=1}^{N} \Gamma(n) \geqslant-1 .
$$

This equation gives conditions for the product averages and therefore for the frequencies of the concurrence of certain values of $A_{\mathbf{a}}^{1}(n), A_{\mathbf{b}}^{2}(n)$ etc. e.g. for $A_{\mathbf{a}}^{1}(n)=$ $+1, A_{\mathbf{b}}^{2}(n)=-1$. These latter frequencies must therefore obey these conditions. Thus we obtain rules or nontrivial inequalities for the frequencies of concurrence of the patients symptoms. Boole calls these rules "conditions of possible experience". In case of a violation, Boole states that then the "evidence is contradictory".

In the opinion of the authors, the term "possible experience" is somewhat of a misnomer. The experimental outcomes have been determined from an experimental procedure in a scientific way and are therefore possible. What may not be possible is the one to one correspondence of Boole's logical elements or variables to the experimental outcomes that the scientist or statistician has chosen. In order to judge precisely where the contradictions arise from, we need to advance 100 years to the work of Vorob'ev on the one side and go back to the meaning of Plato's logic and his rule "aut $P$ aut non $P$ tertium non datur" on the other.

Before doing so, however, we note the following. In this example, we may indeed regard the various $A_{\mathbf{o}}^{l}(n)= \pm 1$ with given indices as the elements of Boole's logic to which the actual experiments can be mapped. As shown by Boole, this is a sufficient condition for the inequality of eq. (4) to be valid. We may in this case also omit all the indices except for those designating the birth place and still will obtain a valid equation that can never be violated:

$$
\left\langle A_{\mathbf{a}} A_{\mathbf{b}}\right\rangle+\left\langle A_{\mathbf{a}} A_{\mathbf{c}}\right\rangle+\left\langle A_{\mathbf{b}} A_{\mathbf{c}}\right\rangle \geqslant-1
$$

The reason is simply that three arbitrary dichotomic variables i.e. variables that assume only two values $( \pm 1$ in our case) must always fulfill eq. (5) no matter what their logical connection to experiments is because we deduce the three products of eq. (5) from sequences of each three measurement outcomes. Note that eq. (5) contains six factors with each birthplace appearing twice and representing then the identical result. Below we will discuss a slightly modified experiment that is much more general and contains six measurement results for the six factors. Before discussing this more general experiment that resembles more clearly EPR experiments we turn now to the findings of Vorob'ev regarding this type of inequalities and Boole's conditions of possible experience.

Obviously the inequality of eq. (3) is non-trivial because based on the fact that the value of all products must be \pm 1 one could only conclude that

$$
\Gamma(n) \geqslant-3 \text {. }
$$

The non-trivial result has the following reason. Boole included into eq. (2) a cyclicity: the outcomes of the first two products determine the outcomes in the third product. Because all outcomes can only be \pm 1 the cyclicity gives rise to eq. (3). Vorob'ev showed precisely 100 years after Boole's original work in a very general way that it is always a combinatorial-topological cyclicity that gives rise to nontrivial inequalities for the mathematical abstractions of experimental outcomes. Boole pointed to the fact that eq. (3) cannot be violated. However, in order to come to that conclusion, the $A_{\mathbf{o}}^{l}(n)$ need, in the first place, to be in a one to one correspondence to Boole's elements of logic that follow the law "aut $A=+1$ aut $A=-1$ tertium non datur". As discussed in the introduction, eternally valid statements about physical experience such as "aut $A=+1$ aut $A=-1$ tertium non datur" can usually not be made when describing the physical world without the use of some coordinates. In the example above these coordinates where the places of birth, the places of examination and the numbering of the exams that were randomly taken. All these coordinates when added need to still allow for a cyclicity in order to make Boole's inequality nontrivial. Therefore, if we have a violation of a non-trivial Boole inequality, then we must conclude that we have not achieved a one to one correspondence of our variables to the elementary eternally true logical variables of Boole and that we need further "coordinates" that will then remove the cyclicity. In order to illustrate all this by a simple example, we consider the following second different statistical investigation of the same disease.

We now let only two doctors, one in Lille and one in Lyon perform the examinations. The doctor in Lille 
examines randomly all patients of types $\mathbf{a}$ and $\mathbf{b}$ and the one in Lyon all of type $\mathbf{b}$ and $\mathbf{c}$ each one patient at a randomly chosen date. Note that in this way, all patients of type $\mathbf{b}$ receive two examinations. The doctors are convinced that neither the date of examination nor the location (Lille or Lyon) has any influence and therefore denote the patients only by their place of birth. After a lengthy period of examination they find:

$$
\Gamma=\left\langle A_{\mathbf{a}} A_{\mathbf{b}}\right\rangle+\left\langle A_{\mathbf{a}} A_{\mathbf{c}}\right\rangle+\left\langle A_{\mathbf{b}} A_{\mathbf{c}}\right\rangle=-3 .
$$

They further notice that the single outcomes of $A_{\mathbf{a}}, A_{\mathbf{b}}$ and $A_{\mathbf{c}}$ are randomly equal to \pm 1 . This latter fact completely baffles them. How can the single outcomes be entirely random while the products are not random at all and how can a Boole inequality be violated hinting that we are not dealing with a possible experience? After lengthy discussions they conclude that there must be some influence at a distance going on and the outcomes depend on the exams in both Lille and Lyon such that a single outcome manifests itself randomly in one city and that the outcome in the other city is then always of opposite sign. Naturally that way they have removed the Vorob'ev cyclicity and we have only the trivial inequality eq. (6) to obey.

However, there are also other ways that remove the cyclicity, ways that do not need to take recourse to influences at a distance. For example we can have a time dependence and a city dependence of the illness as follows. On even dates we have $A_{\mathbf{a}}=+1$ and $A_{\mathbf{c}}=-1$ in both cities while $A_{\mathbf{b}}=+1$ in Lille and $A_{\mathbf{b}}=-1$ in Lyon. On odd days all signs are reversed. Obviously for measurements on random dates we have then the outcome that $A_{\mathbf{a}}, A_{\mathbf{b}}$ and $A_{\mathbf{c}}$ are randomly equal to \pm 1 while at the same time $\Gamma(n)=-3$ and therefore $\Gamma=-3$. We need no deviation from conventional thinking to arrive at this result because now, in order to deal with Boole's elements of logic, we need to add the coordinates of the cities to obtain $\Gamma=\left\langle A_{\mathbf{a}}^{1} A_{\mathbf{b}}^{2}\right\rangle+\left\langle A_{\mathbf{a}}^{1} A_{\mathbf{c}}^{2}\right\rangle+\left\langle A_{\mathbf{b}}^{1} A_{\mathbf{c}}^{2}\right\rangle \geqslant-3$ and the inequality is of the trivial kind because the cyclicity is removed. The date index does not matter for the products since both signs are reversed leaving the products unchanged. However, in actual fact, also this index might have to be included and could be a reason to remove the cyclicity, e.g., $\Gamma=\left\langle A_{\mathbf{a}}^{1}\left(d_{1}\right) A_{\mathbf{b}}^{2}\left(d_{1}\right)\right\rangle+\left\langle A_{\mathbf{a}}^{1}\left(d_{2}\right) A_{\mathbf{c}}^{2}\left(d_{2}\right)\right\rangle+$ $\left\langle A_{\mathbf{b}}^{1}\left(d_{3}\right) A_{\mathbf{c}}^{2}\left(d_{3}\right)\right\rangle \geqslant-3$, where we now have included the fact that the exams of pairs are performed at different dates $d_{1}, d_{2}, d_{3}$.

We note that in connection with EPR experiments and questions relating to interpretations of quantum theory, eqs. (1) and (3) are called Leggett-Garg inequalities and are of the Bell type. It is often claimed that a violation of such inequalities implies that either realism or Einstein locality should be abandoned. As we saw in our counterexample which is both Einstein local and realistic in the common sense of the word, it is the one to one correspondence of the variables to the logical elements of Boole that matters when we determine a possible experience, but not necessarily the choice between realism and Einstein locality. Phrased differently, the question "does the moon shine when we are not looking' is simply too imprecise. Had we given a space-time coordinate for the event that the moon shines we would have expressed an eternal truth of a measurement.

Realism plays a role in the arguments of Bell and followers because they introduce a variable $\lambda$ representing an element of reality and then write

$$
\Gamma=\left\langle A_{\mathbf{a}}(\lambda) A_{\mathbf{b}}(\lambda)\right\rangle+\left\langle A_{\mathbf{a}}(\lambda) A_{\mathbf{c}}(\lambda)\right\rangle+\left\langle A_{\mathbf{b}}(\lambda) A_{\mathbf{c}}(\lambda)\right\rangle \geqslant-1 .
$$

Because no $\lambda$ exists that would lead to a violation except a $\lambda$ that depends on the index pairs $(\mathbf{a}, \mathbf{b}),(\mathbf{a}, \mathbf{c})$ and $(\mathbf{b}, \mathbf{c})$ the simplistic conclusion is that either elements of reality do not exist or they are non-local. The mistake here is that Bell and followers insist from the start that the same element of reality occurs for the three different experiments with three different setting pairs. This assumption implies the existence of the combinatorialtopological cyclicity that in turn implies the validity of a non-trivial inequality but has no physical basis. Why should the elements of reality not all be different? Why should they, for example not include the time of measurement? There is furthermore no reason why there should be no parameter of the equipment involved. Thus the equipment could involve time and setting dependent parameters such as $\lambda_{\mathbf{a}}(t), \lambda_{\mathbf{b}}(t), \lambda_{\mathbf{c}}(t)$ and the functions $A$ might depend on these parameters as well. We refer the reader to refs. $[6,17-20]$ and note that parameters related to the devices of measurement have been discussed already by Wigner [21] but not in connection to the one-to-one correspondence with Boole's logical elements. The possible dependence of these parameters on measurement time or Einstein's space-time prevents the derivation of the Clauser-Horne-Shimony-Holt inequality because outcome independence may be violated, as can be seen directly by using our example of different outcomes for even and odd times in their equations.

Bell revisited from the view of quantum theory. - Consider three spin-1/2 particles that are measured by macroscopic equipment involving three Stern-Gerlach magnets. The wave function of the three particles is not nearer specified and denoted by $\psi_{3}$. If we denote the measurement outcomes at measurement time $n$ for the three particles with the three respective magnet settings by $A_{\mathbf{a}}^{n}\left(\psi_{3}\right), A_{\mathbf{b}}^{n}\left(\psi_{3}\right), A_{\mathbf{c}}^{n}\left(\psi_{3}\right)$, then it is easy to show by the laws of quantum theory that the Boole (Bell) inequality [22]:

$$
\begin{aligned}
\left\langle A_{\mathbf{a}}^{n}\left(\psi_{3}\right) A_{\mathbf{b}}^{n}\left(\psi_{3}\right)\right\rangle & +\left\langle A_{\mathbf{a}}^{n}\left(\psi_{3}\right) A_{\mathbf{c}}^{n}\left(\psi_{3}\right)\right\rangle \\
& +\left\langle A_{\mathbf{b}}^{n}\left(\psi_{3}\right) A_{\mathbf{c}}^{n}\left(\psi_{3}\right)\right\rangle \geqslant-1,
\end{aligned}
$$

is fulfilled and we can conclude that we have dealt with the logical elements of Boole and well-defined probabilities.

If we consider instead six measurements of pairs of particles that are described by the singlet state 
$\psi_{S}$ then we need three different measurement station pairs or one pair of measurement stations at three different measurement times. For simplicity consider three different measurement station pairs that we label with indices $n, m, l$. Correspondingly, we also introduce for the measurement outcomes the symbols $A_{\mathbf{a}}^{n}\left(\psi_{S}\right), A_{\mathbf{b}}^{n}\left(\psi_{S}\right) ; A_{\mathbf{a}}^{m}\left(\psi_{S}\right), A_{\mathbf{c}}^{m}\left(\psi_{S}\right) ; A_{\mathbf{b}}^{l}\left(\psi_{S}\right), A_{\mathbf{c}}^{l}\left(\psi_{S}\right)$. Then quantum theory tells us that for certain magnet settings we may have

$$
\begin{aligned}
\left\langle A_{\mathbf{a}}^{n}\left(\psi_{S}\right) A_{\mathbf{b}}^{n}\left(\psi_{S}\right)\right\rangle & +\left\langle A_{\mathbf{a}}^{m}\left(\psi_{S}\right) A_{\mathbf{c}}^{m}\left(\psi_{S}\right)\right\rangle \\
& +\left\langle A_{\mathbf{b}}^{l}\left(\psi_{S}\right) A_{\mathbf{c}}^{l}\left(\psi_{S}\right)\right\rangle<-1
\end{aligned}
$$

and we have a violation of an inequality that resembles the Bell type. In this case, however, this does not surprise us because as long as we have no cyclicity in the expressions of eq. (10), we obtain only a trivial Boole inequality and as far as Boole's or Kolmogorov's probability are concerned the right-hand side of eq. (10) might as well be -3 . Note that the attachment of space-time indices to the variables that provide a characterization of the experiments in addition to observations such as the magnet settings always permit a removal of any cyclicity. Quantum theory does not have any concerns about the indices $n, m, l$ because quantum theory is careful not to assign any meaning to the single outcomes and therefore does not rely on or need a sample space or probability space.

A probability as in the frameworks of Boole or Kolmogorov is thus not defined in quantum theory because quantum theory does not define any relations of its framework to single logical elements or elementary events and therefore also cannot provide a measure to general sets or subsets of such elements or events. What is defined in quantum theory are long-term averages and these may be related in a variety of ways to the actual logical elements of a theory. The probability amplitude just carries with it all the possibilities that may actually be realized in a set of data, that is all the possibilities that may be realized as a sample space. For an actual sample space to be realized other choices must be made that, in principle, have nothing to do with the quantum particles that are measured but only with the macroscopic equipment that is brought into a certain setting for the purpose of measurement. These other choices may again involve sample spaces and probability spaces that together with the measurement outcomes related to quantum particles may form complex stochastic processes.

Quantum theory predicts the long-term averages of these stochastic processes but does not attempt to unify these processes into one common stochastic process. The Born rule thus attaches positive values to measurement outcomes that are related to certain measurements and preparations and defines in this way what one could call a pre-measure. For all well-defined macroscopic equipment arrangements this pre-measure can be turned into a probability measure with different experimental sequences corresponding, in principle, to different probability measures.
Whether or not these different measures and sample spaces can be unified is a matter of characterization. If no unification is possible, as would be indicated by a violation of a Boole (Bell) inequality, then one needs further detail in the characterization of variables in order to remove the cyclicity. That may be achieved both in an Einstein local way or in a non-local fashion. As we saw above, EPR experiments always permit extended characterization by Einstein's space-time and corresponding avoidance of cyclicity. Non-local characterizations that avoid cyclicity are also always possible but not necessary. The only alternative to the above is to abandon realism (whatever we mean by this word) altogether. The examples (counterexamples) with the patient-investigations and the relation of these examples to EPR experiments prove, at least in the opinion of these authors, that neither realism nor Einstein locality need be abandoned because of a violation of Bell's inequalities.

\section{REFERENCES}

[1] Einstein A., Podolsky A. and Rosen N., Phys. Rev., 47 (1935) 777.

[2] Bohm D. and Aharonov Y., Phys. Rev., 108 (1957) 1070.

[3] Bell J., Physics, 1 (1964) 195.

[4] Boole G., Philos. Trans. R. Soc. London, 152 (1862) 225.

[5] Leggett A. J. and Garg A., Phys. Rev. Lett., 9 (1985) 857.

[6] Hess K. and Philipp W., Proc. Natl. Acad. Sci. U.S.A., 98 (2001) 14228.

[7] ACCARDI L., Some loopholes to save quantum nonlocality, in Foundations of Probability and Physics-3, edited by Khrennikov A., AIP Conf. Proc., 750 (2005) 21.

[8] Rastal P., Phys. Lett., 86A (1981) 85.

[9] Fine A., Phys. Rev. Lett., 48 (1982) 291.

[10] Nieuwenhuizen T. M., Mehmani B., ŠpičKA V., Aghdami M. J. and Khrennikov A. Y., Beyond the Quantum (World Scientific, Singapore) 2007.

[11] Khrennikov A., Theor. Math. Phys., 157 (2008) 1448.

[12] Adenier G. and Khrennikov A. Y., J. Phys. B: At. Mol. Opt. Phys., 40 (2007) 131.

[13] Aspect A., Grangier P. and Roger G., Phys. Rev. Lett., 49 (1982) 91.

[14] Breuer H. and Petruccione F., Open Quantum Systems (Oxford University Press, Oxford) 2002.

[15] Feller W., An Introduction to Probability Theory and its Applications, Vol. 1 (Wiley \& Sons, New York) 1968.

[16] Vorob'ev N., Theor. Probab. Appl., 7 (1962) 147.

[17] Fine A., Synthese, 50 (1982) 279.

[18] De Raedt K., Keimpema K., De Raedt H., Michielsen K. and Miyashita S., Eur. Phys. J. B, 53 (2006) 139.

[19] Zhao S., De Raedt H. and Michielsen K., Found. Phys., 38 (2008) 322.

[20] Khrennikov A. Y., Contextual Approach to Quantum Formalism (Springer, Berlin) 2009.

[21] Wigner E. P., Am. J. Phys., 38 (1970) 1005.

[22] De Raedt H., Hess K. and Michielsen K., http://arxiv.org/abs/0901.2546. 